\title{
Neue Rechtslage \\ bei Suchterkrankungen
}

\section{Marco Weiss}

Dr. iur., Reetz Sohm Rechtsanwälte, Küsnacht ZH

Die Diagnose der Suchterkrankungen hat bisher grundsätzlich keinen sozialversicherungsrechtlichen Rentenanspruch begründen lassen. In seiner alten Rechtsprechung ging das Bundesgericht davon aus, dass Suchterkrankungen von der betroffenen Person «überwunden» werden können und daher in der Regel keine Sozialversicherungsleistungen geschuldet seien. Mit seiner aktuellen Rechtsprechung distanziert sich das höchste Gericht der Schweiz von dieser Sichtweise und verlangt, dass Suchterkrankungen einem strukturierten Beweisverfahren zu unterziehen seien.

Wenn eine Person unter einer Suchterkrankung (Alkohol, Medikamente etc.) leidet, konnte sie nach der bisherigen ständigen bundesgerichtlichen Rechtsprechung keinen Anspruch auf Sozialversicherungsleistungen geltend machen. Aufgrund einer «Überwindbarkeitsvermutung» ging das Bundesgericht über Jahrzehnte davon aus, dass der Gesundheitsschaden der betroffenen Person aufgrund einer Abhängigkeit selbst verursacht worden und diese im Rahmen ihrer Schadensminderungspflicht selbst dafür verantwortlich sei, von der Abhängigkeit loszukommen. Diese «Selbstüberwindung» erscheine möglich und zumutbar.

Aufgrund deutlicher Kritik aus der medizinischen Literatur hat das Bundesgericht diese Rechtsprechung einer Prüfung unterzogen und setzt bei Abhängigkeitserkrankungen - wie bei somatischen Schmerzstörungen und psychischen Gesundheitsleiden - neu auf ein strukturiertes Beweisverfahren.

\section{Frühere Rechtslage}

Nach bisheriger und langjähriger höchstrichterlicher Rechtsprechung führten Suchterkrankungen als solche nicht zu einer rentenbegründenden Invalidität. Sie sind sozialversicherungsrechtlich erst dann relevant geworden, wenn sie eine Krankheit oder einen Unfall bewirkt haben, in deren Folge ein körperlicher oder geistiger, die Erwerbsfähigkeit beeinträchtigender Gesundheitsschaden eingetreten war, oder wenn sie selber Folge eines körperlichen oder geistigen Gesundheitsschadens waren, dem Krankheitswert zugesprochen wurde. Ein invalidisierender psychischer Gesundheitsschaden fehlte demgegenüber, wo in der
Begutachtung im Wesentlichen nur Befunde erhoben worden sind, welche in der Sucht ihre hinreichende Erklärung gefunden haben [1].

Diese genannte Rechtsprechung ging davon aus, dass die süchtige versicherte Person ihren Zustand selbst verschuldet habe. Bei pflichtgemässer Sorgfalt hätte sie die schädlichen Auswirkungen des Substanzkonsums - wenngleich möglicherweise nicht von Anfang an, so doch jedenfalls früh und klar genug - erkennen können und sie abwenden bzw. der Heilung zuführen müssen [2]. Mit anderen Worten ausgedrückt, ging man von der Fiktion einer willentlichen Vermeid- bzw. Überwindbarkeit der Sucht aus [3].

Rechtlich kam darin eine eigentliche Fiktion der willentlichen Vermeid- bzw. Überwindbarkeit der Sucht an sich, und folglich auch der Überwindbarkeit der dadurch verursachten Erwerbsunfähigkeit, zum Ausdruck [4].

Diese Rechtsprechung ist vor allem aus medizinischer Warte kritisiert worden: Ein Suchtmittelentzug sei nach medizinischem Kenntnisstand keineswegs in jedem Fall als zumutbar oder ergebnisorientiert als beste Lösung im Sinne der Schadensminderung anzusehen, und etwaige Funktionseinbussen, Therapiemöglichkeiten und -ergebnisse seien individuell in hohem Masse unterschiedlich [5]. Grundsätzlich sei auch bei Suchterkrankungen eine indikatorengestützte Abklärung im Einzelfall durchzuführen [6].

\section{Aktuelle Rechtslage}

In seiner neuen Rechtsprechung geht das Bundesgericht davon aus, dass die oben genannte «Überwind- 
barkeitsvermutung» bei Suchtkrankheiten nicht mehr getragen werden könne:

Die Medizin definiere das Abhängigkeitssyndrom als eine Gruppe von Verhaltens-, kognitiven und körperlichen Phänomenen, die sich nach wiederholtem Gebrauch von psychotropen Substanzen entwickeln würden. Typischerweise bestehen ein starker Wunsch, die Substanz einzunehmen, eine verminderte Kontrolle über ihren Konsum und ein anhaltender Substanzgebrauch trotz schädlicher Folgen. Dem Substanz-

\section{Tabelle 1: Kriterien nach ICD-10 [10].}

1 Starkes Verlangen (Craving) oder eine Art Zwang, die Substanz zu konsumieren.

2 Verminderte Kontrolle über den Substanzgebrauch, d.h. über Beginn, Beendigung oder die Menge des Konsums, deutlich daran, dass oft mehr von der Substanz oder über einen längeren Zeitraum konsumiert wird als geplant, oder an dem anhaltenden Wunsch oder an erfolglosen Versuchen, den Substanzkonsum zu verringern oder zu kontrollieren.

3 Ein körperliches Entzugssyndrom (siehe F1x.3 und F1x.4), wenn die Substanz reduziert oder abgesetzt wird, mit den für die Substanz typischen Entzugssymptomen oder auch nachweisbar durch den Gebrauch derselben oder einer sehr ähnlichen Substanz, um Entzugssymptome zu mildern oder zu vermeiden.

4 Toleranzentwicklung gegenüber den Wirkungen der Substanz. Für eine Intoxikation oder um den gewünschten Effekt zu erreichen, müssen grössere Mengen der Substanz konsumiert werden, oder es treten bei fortgesetztem Konsum derselben Menge deutlich geringere Effekte auf.

5 Einengung auf den Substanzgebrauch, deutlich an der Aufgabe oder Vernachlässigung anderer wichtiger Vergnügen oder Interessensbereiche wegen des Substanzgebrauchs; oder es wird viel Zeit darauf verwandt, die Substanz zu bekommen, zu konsumieren oder sich davon zu erholen.

6 Anhaltender Substanzgebrauch trotz eindeutig schädlicher Folgen (siehe F1x.1), deutlich an dem fortgesetzten Gebrauch, obwohl der Betreffende sich über die Art und das Ausmass des Schadens bewusst ist oder bewusst sein könnte.

Tabelle 2: Kriterien nach DSM-5 [12]

1 Konsum häufig in grösseren Mengen oder über einen längeren Zeitraum als beabsichtigt.

2 Anhaltender Wunsch oder erfolglose Versuche, den Substanzkonsum zu beenden oder zu kontrollieren.

3 Hoher Zeitaufwand, um die Substanz zu beschaffen, zu konsumieren oder sich von ihren Wirkungen zu erholen.

$4 \quad$ Intensives Verlangen oder Drang nach der Substanz (Craving).

5 Wiederholter Substanzkonsum führt zu wiederholtem Versagen bei der Erfüllung von Verpflichtungen bei der Arbeit, in der Schule oder im Haushalt.

$6 \quad$ Fortgesetzter Substanzkonsum trotz andauernder oder wiederkehrender sozialer oder zwischenmenschlicher Probleme, die durch die Wirkungen der Substanz verursacht oder verschärft werden.

$7 \quad$ Wichtige soziale, berufliche oder Freizeitaktivitäten werden aufgrund des Substanzkonsums aufgegeben oder eingeschränkt.

8 Wiederholter Konsum in Situationen, in denen eine körperliche Gefährdung besteht.

9 Fortgesetzter Konsum trotz Kenntnis eines anhaltenden oder wiederkehrenden körperlichen oder psychischen Problems, das wahrscheinlich durch die Substanz verursacht wurde oder verstärkt wird.

10 Toleranzentwicklung, definiert durch eines der folgenden Kriterien: Verlangen nach ausgeprägter Dosissteigerung, um einen Intoxikationszustand oder einen erwünschten Effekt herbeizuführen; oder deutlich verminderte Wirkung bei fortgesetztem Konsum derselben Menge der Substanz.

11 Bei Zurückgehen der Blut- oder Gewebekonzentration treten Entzugssymptome auf. gebrauch werde der Vorrang vor anderen Aktivitäten und Verpflichtungen gegeben. Es entwickeln sich eine Toleranzerhöhung und manchmal ein körperliches Entzugssyndrom [7]. Aus medizinischer Sicht handle es sich mithin klar um ein krankheitswertiges Geschehen, dessen funktionelle Auswirkungen sich mit einem hypothetischen Substanzentzug nicht ohne Weiteres zurückbilden. Im Übrigen seien auch die $\mathrm{Zu}$ mutbarkeit und die Sinnhaftigkeit eines solchen Entzugs gerade bei langjährigen Verläufen nicht in jedem Fall zu bejahen [8]. Die Diagnose eines Abhängigkeitssyndroms bedinge nach ICD-10 konkret das Bestehen von drei oder mehr der nachfolgenden Kriterien während mindestens einem Monat oder wiederholt innerhalb von zwölf Monaten (siehe Tabelle 1 [9]).

Im Wesentlichen deckungsgleich definiere der DSM-5 (Diagnostisches und Statistisches Manual Psychischer Störungen, fünfte Auflage) die «Substanzkonsumstörungen", wobei innerhalb eines Zeitraums von zwölf Monaten wenigstens zwei der folgenden elf Diagnosekriterien vorliegen müssen (siehe Tabelle 2 [11]).

Aus den gesagten Ausführungen betreffend ICD-10 und DSM-5 resultiere, dass die willentliche Natur des fortgesetzten Substanzkonsums indes bei Vorliegen eines Abhängigkeitssyndroms gerade nicht in jedem Fall vorbehaltlos bejaht werden könne. Diagnosekriterium seien nämlich bei beiden insbesondere der anhaltende Wunsch oder erfolglose Versuche, den Substanzkonsum $\mathrm{zu}$ beenden, $\mathrm{zu}$ verringern oder $\mathrm{zu}$ kontrollieren [13].

Als Konsequenz sei aus höchstrichterlicher Sicht künftig davon auszugehen, dass - fachärztlich einwandfrei diagnostizierten - Abhängigkeitssyndromen bzw. Substanzkonsumstörungen nicht zum Vornherein jede invalidenversicherungsrechtliche Relevanz abgesprochen werden könne [14]. Nachvollziehbar diagnostizierte Abhängigkeitssyndrome bzw. Substanzkonsumstörungen seien zukünftig grundsätzlich als invalidenversicherungsrechtlich beachtliche (psychische) Gesundheitsschäden in Betracht zu ziehen [15]. Zur Beurteilung eines sozialversicherungsrechtlichen IV-Anspruchs müsse künftig wie bei psychischen Erkrankungen und somatischen Schmerzstörungen ein strukturiertes Beweisverfahren durchgeführt werden [16]: Im Rahmen des strukturierten Beweisverfahrens könne und müsse insbesondere dem Schweregrad der Abhängigkeit im konkreten Einzelfall Rechnung getragen werden. Diesem komme nicht zuletzt deshalb Bedeutung zu, weil bei Abhängigkeitserkrankungen wie auch bei anderen psychischen Störungen - oft eine Gemengelage aus krankheitswertiger Störung und psychosozialen sowie soziokulturellen Faktoren vorliege. Letztere seien selbstverständlich auch bei Abhängig- 
keitserkrankungen auszuklammern, wenn sie direkt negative funktionelle Folgen zeitigen würden [17].

\section{Fazit}

Die dargestellte Rechtsprechung verdeutlicht, dass das höchste Gericht der Schweiz vermehrt dazu übergeht, die Beurteilung von sozialversicherungsrechtlichen Ansprüchen bei komplexen Gesundheitsschädigungen einem strukturierten Beweisverfahren zu unterziehen: Dieses wird seit dem Jahr 2015 bei somatischen Schmerzstörungen, seit 2017 bei sämtlichen psychischen Erkrankungen und seit Juli 2019 auch bei Suchterkrankungen angewendet. Damit distanziert sich das Bundesgericht von der beschriebenen (diagnoserelevanten) Überwindbarkeitsvermutung und macht sozialversicherungsrechtliche Ansprüche von mehreren Faktoren, insbesondere der Einschränkung des funktionellen Schweregrades (Ausprägung der diagnoserelevanten Befunde, Behandlungs- und Eingliederungserfolg oder - resistenz) und der Konsistenz des Gesundheitsschadens, abhängig.

Diese neue Rechtsprechung für Suchterkrankungen ist zu begrüssen, weil sie - wie bereits oben beschrieben auf medizinischen Erkenntnissen (ICD-10 und DSM-5) beruht und nicht allein auf einer rechtlich fixierten diagnoserelevanten Überwindbarkeitsfiktion. Im Zuge der neuen Rechtslage bei Suchterkrankungen hat das Bundesgericht diese in seiner Folgerechtsprechung auch konzise angewandt:

- im Falle eines Arztes, der aufgrund einer Alkoholkrankheit nicht mehr als Neurochirurg arbeiten konnte [18];

- bei der Beurteilung eines Abhängigkeitssyndroms (Alkohol) und weiterer psychischer Beschwerden (rezidivierende Depression und Persönlichkeitsstörung) eines Informatik-Beraters [19].

\section{Das Wichtigste in Kürze}

- Suchterkrankungen führten bisher nicht zu einer rentenbegründenden Invalidität, da die Rechtsprechung von einer willentlichen Vermeid- bzw. Überwindbarkeit der Sucht ausging.

- Suchterkrankungen sind sozialversicherungsrechtlich erst dann relevant geworden, wenn sie eine Krankheit oder einen Unfall bewirkt haben, in deren Folge ein körperlicher oder geistiger, die Erwerbsfähigkeit beeinträchtigender Gesundheitsschaden eingetreten war, oder wenn sie selber Folge eines körperlichen oder geistigen Gesundheitsschadens waren, dem Krankheitswert zugesprochen wurde.

- Aufgrund deutlicher Kritik aus der medizinischen Literatur hat das Bundesgericht diese Rechtsprechung einer Prüfung unterzogen und setzt bei Abhängigkeitserkrankungen - wie bei somatischen Schmerzstörungen und psychischen Gesundheitsleiden - neu auf ein strukturiertes Beweisverfahren.
Literatur

1 BGE 124 V 265 E. 1c; 99 V 28 E. 2; Urteil des Bundesgerichts 9C $724 / 2018$ vom 11. Juli 2019 E. 4.1.

2 EVGE 1962101 E. 3; Urteil des Bundesgerichts 9C_724/2018 vom 11. Juli 2019 E. 4.2.

3 Vgl. BGE 139 V 547 E. 5.1; Urteil des Bundesgerichts 9C 724/2018 vom 11. Juli 2019 E. 4.2 .

4 Urteil des Bundesgerichts 9C_724/2018 vom 11. Juli 2019 E. 4.2.

5 Liebrenz M, et al. Das Suchtleiden bzw. die Abhängigkeitserkrankungen - Möglichkeiten der Begutachtung nach BGE $141 \mathrm{~V} 281$ [=9C_492/2014]. SZS 2016. S. 12 ff., S. 22 und S. 30 ff.

6 Liebrenz M, et al. Das Suchtleiden bzw. die Abhängigkeitserkran kungen - Möglichkeiten der Begutachtung nach BGE $141 \mathrm{~V} 281$ [=9C 492/2014]. SZS 2016. S. 28 f. und S. 32; Liebrenz M, Uttinger U, Ebner G. Sind Abhängigkeitserkrankungen aus höchstrichterlicher Sicht [weiterhin] nicht mit anderen psychischen Störungen [z.B. somatoformen Störungen] vergleichbar? Eine Urteilsbesprechung von BGE 8C_582/2015 im Lichte der theoretischen Anwendbarkeit des ergebnisoffenen, strukturierten Beweisverfahrens. SZS 2016. S. $96 \mathrm{ff}$. und S. $99 \mathrm{f}$.

7 Dilling H, Freyberger HJ. Taschenführer zur ICD-10-Klassifikation psychischer Störungen. 8. A. Bern: Hogrefe; 2016. S. 76 ff.; Daeppen JB. Die therapeutische Begleitung von Suchtpatienten. Schweiz Med Forum. 2016;16(18-19):423-6. S. 424; Urteil des Bundesgerichts 9C_724/2018 vom 11. Juli 2019 E. 5.2.1.

8 Liebrenz M, et al. Das Suchtleiden bzw. die Abhängigkeitserkrankungen - Möglichkeiten der Begutachtung nach BGE $141 \mathrm{~V} 281$ [=9C 492/2014]. SZS 2016. S. 30 ff.; Urteil des Bundesgerichts 9C_ $724 / 2018$ vom 11. Juli 2019 E. 5.2.1.

9 Dilling H, Freyberger HJ. Taschenführer zur ICD-10-Klassifikation psychischer Störungen. 8. A. Bern: Hogrefe; 2016. S. 77 f.; Urteil des Bundesgerichts 9C_724/2018 vom 11. Juli 2019 E. 5.2.1.

10 Dilling H, Freyberger HJ. Taschenführer zur ICD-10-Klassifikation psychischer Störungen. 8. A. Bern: Hogrefe; 2016. S. 77 f.; Urteil des Bundesgerichts 9C_724/2018 vom 11. Juli 2019 E. 5.2.1.

11 Falkai P, et al. [Ed.]. Diagnostisches und Statistisches Manual Psychischer Störungen DSM-5. Deutsche Ausgabe. Bern: Hogrefe; 2015. S. 661 ff. und S. 663 f.; Urteil des Bundesgerichts 9C_724/2018 vom 11. Juli 2019 E. 5.2.2.

12 Falkai P, et al. [Ed.]. Diagnostisches und Statistisches Manual Psychischer Störungen DSM-5. Deutsche Ausgabe. Bern: Hogrefe; 2015. S. 661 ff. und S. 663 f.; Urteil des Bundesgerichts 9C_724/2018 vom 11. Juli 2019 E. 5.2.2.

13 Urteil des Bundesgerichts 9C 724/2018 vom 11. Juli 2019 E. 5.3.2.

14 Urteil des Bundesgerichts 9C_724/2018 vom 11. Juli 2019 E. 5.3.3.

15 Urteil des Bundesgerichts 9C_724/2018 vom 11. Juli 2019 E. 6.

16 Zum Ganzen BGE 143 V 418; 143 V 409 und 141 V 281; Urteil des Bundesgerichts 9C_724/2018 vom 11. Juli 2019 E. 6.

17 Urteil des Bundesgerichts 9C 724/2018 vom 11. Juli 2019 E. 6.3.

18 Urteil des Bundesgerichts 8C_245/2019 vom 16. September 2019.

19 Urteil des Bundesgerichts 9C_334/2019 vom 6. September 2019.

\section{L'essentiel en bref}

- Jusqu'à maintenant, les troubles addictifs n'impliquaient pas une invalidité justifiant le versement d'une rente, car la jurisprudence partait du principe que la dépendance pouvait être évitée ou surmontée par la volonté.

- Les troubles addictifs peuvent désormais être couverts par les assurances sociales à condition d'avoir provoqué une maladie ou un accident qui entraîne une atteinte à la santé physique ou mentale, nuisant à la capacité de gain, ou de résulter eux-mêmes d'une atteinte à la santé physique ou mentale qui a valeur de maladie.

- A la lumière de la critique de la littérature médicale, le Tribunal fédéral a examiné cette jurisprudence et, pour les troubles addictifs (comme pour les douleurs somatoformes et les souffrances psychiques) il s'appuie désormais sur une procédure structurée d'administration des preuves.
Dr. iur. Marco We

marco.weiss[at

reetz-sohm.ch 